\title{
Rapid, High-Resolution Raman Imaging of Pharmaceutical, Biological, and Other Materials with the Thermo Scientific DXRxi.
}

\author{
Robert A. Heintz, Mark H. Wall, and Jennifer L. Ramirez
}

Thermo Fisher Scientific, Madison, WI, USA

Raman spectroscopy is a facile method that can be used to analyze a wide variety of samples, such as materials pertaining to life sciences and healthcare, like pharmaceuticals and biological materials, to high technology nanomaterials such as graphene and carbon nanotubes. Many analytical challenges exist when analyzing the aforementioned samples, such as a requirement for high spatial resolution, difficult and time-consuming sample preparation, as well as the need for rapid answers. Raman spectroscopy, and specifically Raman spectroscopic imaging, offers high resolution, on the order of $1 \mu \mathrm{m}$ or better, minimal sample preparation is required to obtain a Raman spectrum, and Raman spectroscopy can differentiate between compounds on a molecular level that would be indistinguishable on an atomic level. The research presented herein utilizes the Thermo Scientific ${ }^{\mathrm{TM}}$ DXR $^{\mathrm{TM}_{\mathrm{X}}}$ Raman imaging microscope to analyze these types of pharmaceutical sample, biological materials, and more.

Pharmaceutical formulations can contain many different components and are often quite complex. The distribution and morphology of each component can affect the stability and functionality of the final product. Raman spectroscopy is used in this research to detect the type of component, its location, and the amount of component present in two different pharmaceutical formulations a hormone tablet with a single active ingredient present in small quantities and a headache tablet with three active ingredients.

Tibolone is a synthetic hormone that may be present in a pharmaceutical formulation in either of two polymorphs, monoclinic or triclinic [1]. The type of polymorph present in any pharmaceutical formulation may impact the therapeutic properties of the tablet as a whole. An entire tablet of Tibolone was analyzed with the DXRxi microscope. The tablet was $6 \mathrm{~mm}$ in diameter and imaged with $25 \mu \mathrm{m}$ pixel spacing. The Tibolone is present at low concentrations, less than $3 \%$. A peak height analysis was performed on the Raman spectroscopic image, and a profile image was generated showing the location of both polymorphs. Further analysis of the chemical image was accomplished during this research to differentiate between the monoclinic and triclinic polymorph of the active ingredient.

A second application of Raman spectroscopic imaging on pharmaceutical formulations is illustrated in Figure 2, which shows a high resolution, $0.5 \mu \mathrm{m}$-pixel size image of an entire three component headache tablet. This tablet measures $11 \mathrm{~mm}$ in diameter, and required 5.4 million spectra to analyze completely. With the DXRxi microscope, this was a 6 hour analysis. The spectroscopic image was analyzed by Multivariate Curve Resolution (MCR), which determined the size, location, and pure component spectrum of the three active ingredient, acetylsalicylic acid, acetaminophen, and caffeine. Also detected by MCR was the outer tablet coating, which was titanium dioxide.

Raman spectroscopic imaging has also been applied to the field of life sciences, and can be used to evaluate the formation of dental caries and developmental defects in the teeth. The type of carbonate present, A-type or B-type, affect the solubility of the tooth in acidic foods or under conditions wherein bacteria digest food and the local environment becomes acidic. Raman spectroscopy is a non-destructive technique that has been previously used to quantify these carbonates, and provides a significant 
advantage over previous methods of carbonate determination in teeth, which included digestion in acid followed by quantitative measurement of the evolved carbon dioxide [2]. The DXRxi was used to obtain a Raman chemical image of the cross section of a human tooth. Of note is that four different types of tooth material are detected and spatially differentiated: pulp, circumpulpal dentin, dentin, and enamel.

\section{References:}

[1] G. Araujo, et al, J Therm Anal Calorim 102 (2010), p. 233.

[2] V. Zavala-Alonso, et al, Journal of Oral Science 54 (2012), p 93.
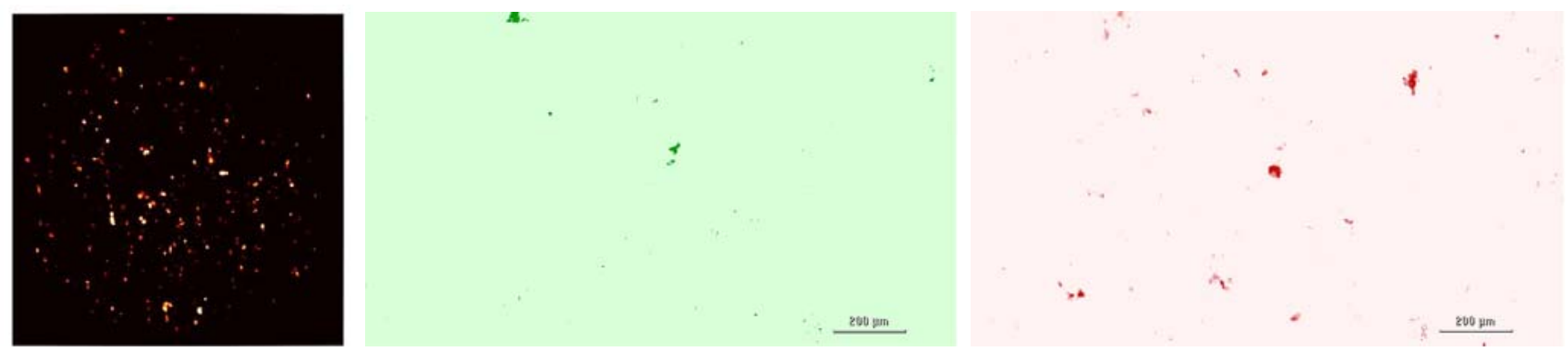

Figure 1. At left, the peak height analysis at $2102 \mathrm{~cm}^{-1}$ of the Tibolone tablet. The bright spots correspond with the spatial location of Tibolone on the tablet. Diameter of the tablet is $6 \mathrm{~mm}$. The center and right images show the locations of the monoclinic and triclinic polymorphs, respectively.

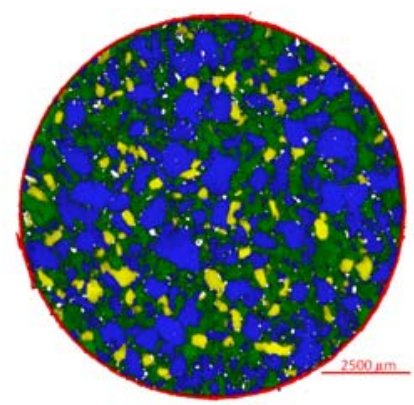

Figure 2. High resolution MCR analysis of the Raman spectroscopic image of an entire three-component headache tablet. Of interest is the presence of the active ingredients as well as the $\mathrm{TiO}_{2}$ outer coating on the tablet. The scale bar in the image is $2500 \mu \mathrm{m}$, and the tablet is $11 \mathrm{~mm}$ in diameter.
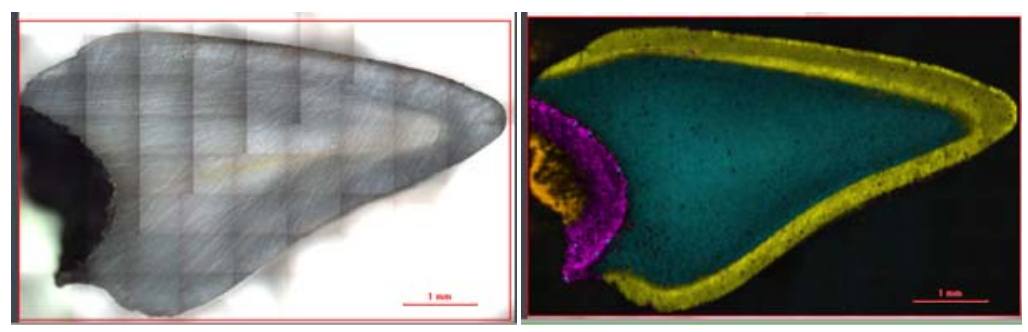

Figure 3. Optical image (left) and Raman spectroscopic image (right) of a human tooth. The presence of the pulp (orange), circumpulpal dentin (purple), dentin (teal), enamel (yellow) can be clearly seen and distinguished from one another. Scale bar is $1 \mathrm{~mm}$ 